\title{
Produtividade da soja correlacionada com a porosidade e a densidade de um Latossolo Vermelho do cerrado brasileiro
}

\author{
Soybean yield correlated with the porosity and the density of a red Latosol of the Brazilian Savanna
}

\author{
Marcelo Andreotti ${ }^{*}$ Morel de Passos e Carvalho' ${ }^{\mathrm{I}}$ Rafael MontanariII \\ Fernanda Carvalho Basso ${ }^{I}$ Cristiano Magalhães Pariz ${ }^{I}$ Mariana Vieira Azenha ${ }^{\mathrm{I}}$ \\ Francine Vercese ${ }^{\mathrm{I}}$
}

\section{RESUMO}

No ano agrícola de 2004/05, na Fazenda Experimental da FE/Unesp-Campus de Ilha Solteira, localizada no Município de Selvíria, Mato Grosso do Sul - (MS), foram estudadas a variabilidade e a dependência espacial entre a macroporosidade (MA), a microporosidade (MI), a porosidade total (PT) e a densidade do solo (DS), em três profundidades, com a produtividade da soja (PG), em sistema plantio direto, sobre um Latossolo Vermelho distroférrico. O objetivo foi pesquisar a variabilidade e as correlações lineares e espaciais entre os atributos da planta e do solo, visando a selecionar um indicador da qualidade física do solo para a produtividade da soja. Foi instalada a malha geoestatística, para a coleta de dados do solo e da planta, contendo 124 pontos amostrais, numa área de $4.000 \mathrm{~m}^{2}$. A correlação linear entre a produtividade de grãos de soja com os atributos do solo estudados foi baixa. Houve correlação espacial direta entre a PG e a MA nas camadas de zero-0,10m e 0,10-0,20m. A macroporosidade do solo (MA1), avaliada na camada de zero$0,10 \mathrm{~m}$, apresentou-se como o melhor indicador da qualidade física do solo quando destinada à avaliação da produtividade de soja, nas condições de plantio direto, em solo de cerrado.

Palavras-chave: compactação do solo, macroporosidade, microporosidade, Glycine max.

\section{ABSTRACT}

The variability and the spatial dependence attributes of the following plant and soil: a) the soybean yield (SY) in no-tillage and irrigated, b) the macroporosity (MA), c) microporosity (MI), d) total porosity (TP), e) and bulk density (BD), in depths of $1(0-0.10 \mathrm{~m}), 2(0.10-0.20 \mathrm{~m})$ and $3(0.20-$ $0.30 \mathrm{~m}$ ), in a Red Latosol of the Engineering college of the São Paulo State University (FE/UNESP) in the Ilha Solteira

\begin{abstract}
campus, located in Selvíria County, Mato Grosso do Sul state, Brazil, were analyzed in the agricultural year of 2004/05. The main goal was to study the variability and the linear and spatial correlations among the attributes (plant and soil), aiming to select an index of soil physical quality with good capacity to represent the soybean yield. A geostatistical grid to collect soil and plant data was installed, with one hundred and twenty four sample points, in an area of $4000 \mathrm{~m}^{2}$. The linear relationship between soybean yield and physical attributes of soil studied was low. So, there was a direct spatial relationship between SY and MA1 (0-0.10m), as well as between SY and MA2 (0.10$0.20 \mathrm{~m})$. The MA1 showed its best index of the soil physical quality, when it was destined to estimate the soybean yield on no-tillage.
\end{abstract}

Key words: soil compactation, macroporosity, microporosity, Glycine max.

\section{INTRODUÇÃO}

A cultura da soja, com destaque econômico na agricultura brasileira, tem se apresentado como umas das grandes alternativas de cultivo quando no sistema plantio direto (SPD). Entretanto, pela pouca diversificação dos sistemas de rotação, normalmente com a soja na primavera/verão e o milho na safrinha, o solo, pela deficitária exploração do sistema radicular dessas culturas, aliado ao tráfego intenso do maquinário, tende à compactação (JOHANN et al., 2004; MELLO FILHO et al., 2006; REICHERT et al., 2009). Como resultado dessa compactação, cada vez mais ocorre o menor crescimento radicular das culturas, com

IDepartamento de Fitossanidade, Engenharia Rural e Solos, Faculdade de Engenharia, Universidade Estadual Paulista (UNESP). Av. Brasil, 56, centro, 15385-000, Ilha Solteira, SP, Brasil. E-mail: dreotti@agr.feis.unesp.br. *Autor para correspondência.

IIFundação Universidade Federal de Mato Grosso do Sul (UFMS), Campus de Chapadão do Sul. Chapadão do Sul, MS, Brasil. 
reflexo na queda da produtividade (TORMENA et al., 1998; FARACO et al., 2008; REICHERT et al., 2009). O solo advém da interação de fatores e processos de formação que variam no decorrer do tempo, da litologia, da topografia, do clima, da atividade biológica e da idade da área a ser estudada. Tal interação transmite uma heterogeneidade, resultando em um sistema dinâmico (TRANGMAR et al., 1985). Assim sendo, esse aumento da heterogeneidade do solo, em sistemas conservacionistas de manejo, criam um ambiente diferente daquele encontrado no sistema convencional, resultante dos efeitos dos resíduos superficiais e da reduzida movimentação. Dessa forma, o mapeamento dos seus atributos físicos, numa área agrícola, é de fundamental importância, tanto para a recomendação de práticas de manejo, como para a avaliação dos efeitos da agricultura sobre a qualidade ambiental.

A análise geoestatística é a ferramenta para avaliar a variabilidade espacial dos atributos estudados e mapeados, por meio da interpolação por krigagem, ou a partir de estimativas de duas variáveis que se correlacionem pela cokrigagem. Elas podem ser descritas como técnicas que minimizam a variância estimada por meio de análise de regressão, levando-se em conta a dependência entre os dados distribuídos no espaço. Dessa forma, as interpolações por krigagem e por cokrigagem estão fortemente associadas aos modelos de semivariogramas ajustados, que podem ser avaliados por meio da validação cruzada (SOARES, 2000).

Muitos estudos relativos à compactação do solo, que utilizam sua densidade como atributo indicador, convergem para o fato de que seu aumento desencadearia, no geral, uma diminuição da produtividade agrícola (ABREU, et al., 2003; FARACO et al., 2008; REICHERT et al., 2009). A porosidade total, por se referir às fases líquida e gasosa do solo, está estreitamente ligada aos processos bioquímicos das plantas e sua produtividade e, de acordo com KIEHL (1979), ela é referida como ideal quando se apresentar com $0,50 \mathrm{~m}^{3} \mathrm{~m}^{-3}$ do seu volume total.

Assim, o objetivo do presente trabalho foi pesquisar a variabilidade e as correlações lineares e espaciais, entre a produtividade da soja e os atributos físicos do solo, visando a selecionar um indicador de qualidade física de correlação com a produtividade da soja.

\section{MATERIAL E MÉTODOS}

A pesquisa foi desenvolvida em área irrigada (pivô central), na Fazenda Experimental, da Faculdade de Engenharia de Ilha Solteira (FE/UNESP), localizada no Município de Selvíria, Mato Grosso do Sul (MS), entre as latitudes de $20^{\circ} 18^{\prime} 05^{\prime \prime} \mathrm{S}$ e $20^{\circ} 18^{\prime} 28^{\prime \prime} \mathrm{S}$ e as longitudes de $52^{\circ} 39^{\prime} 02^{\prime \prime} \mathrm{W}$ e $52^{\circ} 40^{\prime} 28^{\prime} \mathrm{W}$. Sua precipitação média anual é de $1300 \mathrm{~mm}$, enquanto que a temperatura média é de $23,7^{\circ} \mathrm{C}$. O tipo climático é $\mathrm{Aw}$, segundo Koeppen, caracterizado como tropical úmido com estação chuvosa no verão e seca no inverno. O solo, no qual a malha experimental foi instalada, classificado segundo a Embrapa (2006), é um LATOSSOLO VERMELHO Distroférrico típico argiloso, sendo sua área cultivada em sistema plantio direto há cinco anos.

A soja da cultivar 'BRS-133' foi semeada em 11/11/04, em espaçamento de $0,45 \mathrm{~m}$ entre linhas e 16 sementes/metro, adubada na semeadura com $250 \mathrm{~kg}$ da fórmula 8-28-16. Após 30 dias da emergência das plantas, foram definidas as direções dos eixos cartesianos da malha geoestatística, numa área entre dois terraços agrícolas. Assim, o eixo $x$ foi estabelecido em nível, enquanto que o $y$ ficou no sentido do declive, sendo constituída de 124 pontos, distribuídos numa área de $4000 \mathrm{~m}^{2}(40 \mathrm{mx} 100 \mathrm{~m})$, num espaçamento de $10 \mathrm{~m}$ na grande malha e 5,0 e de $1,67 \mathrm{~m}$ na de refinamento. $\mathrm{O}$ objetivo desse refinamento foi de detectar alcances da dependência espacial para espaçamentos menores que os da grande malha.

As áreas úteis utilizadas para a coleta de dados (solo e planta) ficaram estabelecidas no entorno de cada ponto amostral, da seguinte forma: a) na grande malha, com $3,24 \mathrm{~m}^{2}$; e b) nos espaçamentos de refinamento com $1,82 \mathrm{~m}^{2}$. No dia 29/03/05, foram colhidas as plantas no entorno do ponto amostral para determinação da produtividade de grãos (PG), corrigida para $13 \%$ de umidade.

Após a colheita da soja, foram efetuadas as coletas de solo em cada um dos 124 pontos da malha, sendo tomadas amostras indeformadas do solo (anéis volumétricos), individualmente coletadas nas profundidades $1(0-0,10 \mathrm{~m}), 2(0,10-0,20 \mathrm{~m})$ e $3(0,20$ $0,30 \mathrm{~m})$. Assim, foram coletados os seguintes atributos do solo: a) macroporosidade (MA1, MA2, MA3); b) microporosidade (MI1, MI2, MI3); c) porosidade total (PT1, PT2, PT3); e d) densidade do solo (DS1, DS2, DS3). Todos os atributos originaram-se de amostras indeformadas, coletadas em anéis volumétricos (EMBRAPA, 1997).

$\mathrm{Na}$ análise estatística, realizou a análise descritiva inicial dos atributos, com a utilização do software estatístico SAS (SCHLOTZHAVER \& LITTELL, 1997). Posteriormente, realizou-se a identificação de outliers, de acordo com o gráfico de ramos e folhas. Dessa forma, efetuou-se a substituição desses valores pelo valor médio dos circunvizinhos 
contidos na malha geoestatística. Para testar a hipótese de normalidade ou de lognormalidade dos atributos (ATR), realizou-se o teste de SHAPIRO \& WILK (1965) (estatística W), a 1\% de probabilidade. De acordo com o referido teste, a estatística $\mathrm{W}$ testa a hipótese nula, a qual julga ser amostra proveniente de uma população com distribuição normal. No caso de dados transformados na forma logarítmica ( $\mathrm{y}=\log$ ATR), W testa a hipótese nula de que os valores $\mathrm{y}_{\mathrm{i}}$ provêm de uma distribuição normal, ou seja, os dados não transformados $\left(\mathrm{ATR}_{\mathrm{i}}\right)$ ajustam-se a uma distribuição lognormal. Porém, na tabela de análise descritiva inicial, os valores da média, da mediana, do mínimo e do máximo foram retrotransformados pelo uso de $\mathrm{ATR}=1000^{(\log \mathrm{ATR})}$ e sucedidos das letras "d", com o objetivo de que, após anamorfose, seus dados não resultassem em valores menores do que um. A análise estatística também incluiu a análise da correlação linear (simples e múltipla) entre eles. Assim, procurou-se selecionar aqueles de maior correlação linear, sendo, portanto, candidatos a apresentar semivariograma cruzado e a consequente cokrigagem. Além disso, conjuntamente para todas as camadas estudadas do solo, foi efetuada a regressão linear múltipla entre a variável dependente da planta (PG) e as independentes do solo, objetivando selecionar aquelas que proporcionariam as melhores relações entre causa e efeito, avaliadas pelo aumento do $\mathrm{r}^{2}$. Por fim, a análise estatística também incluiu a análise geoestatística, na qual, para cada atributo, foi analisada a dependência espacial pelo cálculo do semivariograma, com o pacote computacional $\mathrm{GS}^{+}$ (2004). Foram ajustados semivariogramas simples a todos os atributos estudados. Posteriormente, ajustaram-se semivariogramas cruzados entre os atributos. O objetivo foi definir os pares de melhor representatividade, isto é, qual atributo, tido como variável secundária, apresentou melhor correlação espacial explicativa do atributo tido como variável primária. Portanto, os ajustes dos semivariogramas (simples e cruzados), em função de seus modelos, foram efetuados pela seleção inicial de: a) a menor soma dos quadrados dos desvios (SQR); b) o maior $\mathrm{r}^{2}$ e c) o maior avaliador da dependência espacial (ADE). A decisão final do modelo que representou o ajuste foi realizada por meio da validação cruzada, assim como também para a definição do tamanho da vizinhança que proporcionou a melhor rede de krigagem e/ou cokrigagem.

\section{RESULTADOS E DISCUSSÃO}

$\mathrm{Na}$ tabela 1, a PG apresentou alta variabilidade, enquanto que, para a MA, nas três camadas, foi muito alta. Já para os atributos MI, PT e
DS, a variabilidade foi baixa, ficando em consonância com os dados de CARVALHO et al. $(2002,2003)$ e FARACO et al. (2008), com exceção à microporosidade que para estes foi média, assim como em SOUZA et al. (2001), que verificaram média variabilidade para a macroporosidade. Segundo FREDDI et al. (2006), o manejo do solo é um fator decisivo para o aumento da sua heterogeneidade. Assim, a macroporosidade por ser um atributo com grande interferência do manejo do solo, com destaque em sistema plantio direto proporcionou os maiores coeficientes de variação. Por outro lado, considerando os demais atributos avaliados, os menores coeficientes de variação constatados nas três camadas atestaram que o sistema plantio direto também pode corroborar o aumento da homogeneidade do solo estudado.

No geral, nas três camadas (Tabela 1), o solo se apresentou compactado, pois, de acordo com KIEHL (1979), a macroporosidade ideal deveria estar acima de $0,100 \mathrm{~m}^{3} \mathrm{~m}^{-3}$, valor atestado também na revisão efetuada por REICHERT et al. (2009), sugerindo tais resultados na implicação de uma baixa produtividade da soja. Entretanto, como a PG média foi de $3270 \mathrm{~kg} \mathrm{ha}^{-1}$, ficou pouco acima do limite superior de $3000 \mathrm{~kg} \mathrm{ha}^{-1}$ preconizado por FAHL et al. (1998) para as condições do Estado de São Paulo. Como a área de cultivo foi irrigada e adubada com base na análise química inicial do solo, a apreciável produtividade foi devida, provavelmente, a essas condições favoráveis.

Já com relação à densidade do solo, seus valores médios denotaram uma tendência linear decrescente, em relação ao aumento em profundidade do solo, indicando que a camada superior se encontrava com maior compactação. Tal fato deveu-se ao SPD, no qual a camada superficial normalmente sofre maior ação do tráfego de máquinas, ficando portanto diferente aos resultados obtidos por SOUZA et al. (2001), CARVALHO et al. (2002) e FARACO et al. (2008), cujas densidades apresentaram aumentos em profundidade do solo, provavelmente em razão da redução do seu teor de MO, em sistema de preparo convencional. Os valores de densidade encontrados no presente ficaram muito próximos dos níveis críticos indicados por KIEHL (1979), situados ao redor de $1,70 \mathrm{~kg} \mathrm{dm}^{-3}$, como capazes de afetar o crescimento das plantas. Entretanto, na revisão efetuada por REICHERT et al. (2009), há citação de que valores acima de $1,21 \mathrm{~kg} \mathrm{dm}^{-3}$ poderiam restringir a elongação radicular da soja e que valores entre 1,36 e $1,68 \mathrm{~kg} \mathrm{dm}^{-3}$ poderiam afetar negativamente, tanto o crescimento, quanto a produtividade da soja em solos argilosos tropicais.

A porosidade total do solo (Tabela 1) apresentou valores crescentes em profundidade e 
Tabela 1 - Análise descritiva inicial da produtividade de grãos de soja (PG) e de alguns atributos físicos (macoporosidade - MA, microporosidade - MI, porosidade total - PT e densidade do solo - DS) de um Latossolo Vermelho Distroférrico da Fazenda de Ensino, Pesquisa e Extensão - FE/Unesp, Selvíria - MS.

\begin{tabular}{|c|c|c|c|c|c|c|c|c|c|c|}
\hline \multirow{2}{*}{ Atributos } & \multirow{2}{*}{ média } & \multirow{2}{*}{ mediana } & \multicolumn{2}{|c|}{--------Valores--------- } & \multirow{2}{*}{$\begin{array}{l}\text { Desvio } \\
\text { padrão }\end{array}$} & \multicolumn{3}{|c|}{---------Coeficientes--------- } & \multicolumn{2}{|c|}{ Probabilidade do teste ${ }^{(\mathrm{a})}$} \\
\hline & & & Mínima & máximo & & $\begin{array}{c}\text { variação } \\
(\%)\end{array}$ & curtose & assimetria & $\mathrm{Pr}<\mathrm{W}$ & $\mathrm{DF}^{(\mathrm{a})}$ \\
\hline \multicolumn{11}{|c|}{ - } \\
\hline PG & 3270 & 3097 & 1209 & 5469 & 993 & 30,4 & $-0,815$ & 0,320 & $5,9.10^{-3}$ & $\mathrm{TN}$ \\
\hline MA1 & 0,056 & 0,048 & 0,010 & $\begin{array}{l}---m a c r o l \\
0,139\end{array}$ & $\begin{array}{r}\text { rosidade }(\mathrm{m} \\
3,080.10^{-2}\end{array}$ & $\begin{array}{c}\left.\mathrm{m}^{-3}\right)----- \\
55,0\end{array}$ & $-0,762$ & 0,525 & $1.10^{4}$ & IN \\
\hline MA2 & 0,054 & 0,050 & 0,005 & 0,116 & $3,001.10^{-2}$ & 55,6 & $-1,107$ & 0,191 & $3.10^{4}$ & IN \\
\hline MA3 & 0,061 & 0,061 & 0,010 & 0,137 & $3,149.10^{-2}$ & 51,7 & $-0,821$ & 0,297 & $2,4.10^{4}$ & IN \\
\hline & & & & ---micro & rosidade (m & $\left.m^{-3}\right)$ & -..- & & & ----- \\
\hline MI1 & 0,370 & 0,364 & 0,312 & 0,452 & $3,5.10^{-2}$ & 9,4 & $-0,774$ & 0,420 & - & IN \\
\hline MI2 & 0,372 & 0,369 & 0,314 & 0,454 & $3,4.10^{-2}$ & 9,2 & $-0,548$ & 0,481 & - & IN \\
\hline MI3 & 0,384 & 0,382 & 0,306 & 0,456 & $3,3.10^{-2}$ & 8,6 & $-0,615$ & 0,032 & 0,368 & $\mathrm{NO}$ \\
\hline & & & & ---poros & de total $\left(\mathrm{m}^{3}\right.$ & $\left.m^{-3}\right)-$ & & & & \\
\hline PT1 & 0,425 & 0,425 & 0,365 & 0,502 & $2,8 \cdot 10^{-2}$ & 6,5 & 0,141 & 0,290 & 0,337 & $\mathrm{NO}$ \\
\hline PT2 & 0,426 & 0,423 & 0,368 & 0,499 & $2,7.10^{-2}$ & 6,2 & 0,154 & 0,445 & 0,086 & NO \\
\hline PT3d ${ }^{(b)}$ & 0,443 & 0,440 & 0,386 & 0,524 & $2,6.10^{-2}$ & 1,0 & 0,546 & 0,398 & 0,086 & $\mathrm{LN}$ \\
\hline & & & & -densida & do solo $(\mathrm{kg}$ & $\left.\mathrm{dm}^{-3}\right)$ & & & & . \\
\hline DS1 & 1,517 & 1,515 & 1,319 & 1,647 & $6,8 \cdot 10^{-2}$ & 4,5 & $-0,200$ & $-0,333$ & 0,092 & NO \\
\hline DS2 & 1,491 & 1,486 & 1,332 & 1,630 & $6,4 \cdot 10^{-2}$ & 4,3 & $-0,362$ & 0,091 & 0,467 & NO \\
\hline DS3 & 1,441 & 1,437 & 1,246 & 1,615 & $6,9 \cdot 10^{-2}$ & 4,8 & 0,457 & $-0,091$ & 0,463 & $\mathrm{NO}$ \\
\hline
\end{tabular}

(a) $\mathrm{DF}$ = distribuição de frequência, sendo NO, LN, IN e TN, respectivamente, do tipo normal, lognormal, indeterminado e tendendo à normal; (b) atributo sucedido da letra "d" teve seu valor multiplicado por 1000 e, seguidamente, calculado o valor do respectivo logaritmo decimal; e valores $\mathrm{x}^{*}$ da média, da mediana, do mínimo e do máximo retrotransformados por $\mathrm{x}^{*}=10^{\log \mathrm{x}} / 1000$; camadas de 1 , 2 e 3 correspondem respectivamente a $0,0-0,10 ; 0,10-0,20$ e $0,20-0,30 \mathrm{~m}$.

correlacionados de forma lógica com os valores de densidade, os quais aumentaram substancialmente em superfície, denotando um evidente estado de maior compactação do solo na primeira e na segunda camada, em virtude do SPD. Além do mais, pelos baixos valores de MA, a PT houve maior influência da microporosidade, que, por sua vez, apresentou o mesmo comportamento de aumento de valores em profundidade como na PT.

Das correlações lineares, entre o atributo $\mathrm{PG}$ e os do solo (Tabela 2), aquelas significativas foram para os pares PGXMA1, PGXMA3, PGXMI1 e PGXDS1. Os dois últimos pares (equações 3 e 4 ) indicaram função decrescente entre causa e efeito. Esse fato está de acordo com o estudo de SANTOS et al. (2006), que verificaram a mesma tendência para a densidade do solo, quando correlacionada com a produtividade de grãos de milho. Assim, verificou-se que o aumento da DS e da MI na superfície do solo resultou em menores produtividades de grãos de soja. Já para os dois primeiros pares (PGXMA1 e PGXMA3), houve correlação positiva entre causa e efeito. Assim sendo, o aumento da aeração do solo na superfície e em profundidade proporcionou uma provável melhoria do crescimento radicular e, por conseguinte, aumento de produtividade. A PG, em função dos atributos do solo nos pares supracitados, pôde ser estimada pelas seguintes equações de regressão linear simples:

$\mathrm{PG}=2,736 \cdot 10^{3}+9,523 \cdot 10^{3 * *} . \mathrm{MA} 1$

$\left(\mathrm{r}=0,295^{* *}\right)$

$\mathrm{PG}=2,767 \cdot 10^{3}+8,256 \cdot 10^{3^{* *}} \cdot \mathrm{MA} 3$

$\left(\mathrm{r}=0,262^{* *}\right)$.

$\mathrm{PG}=5,418 \cdot 10^{3}-5,807.10^{3 *} \cdot \mathrm{MI} 1$

$\left(\mathrm{r}=0,204^{*}\right)$.

$\mathrm{PG}=8,208 \cdot 10^{3}-3,257.10^{3^{*}} . \mathrm{DS} 1$

$\left(\mathrm{r}=0,221^{*}\right)$.

e em relação ao atributo $\mathrm{PG}$, quando estabelecido como variável dependente e os do solo como variáveis independentes, foi ajustada a seguinte equação de regressão linear múltipla: 
Tabela 2 - Matriz de correlação linear simples entre a produtividade de grãos de soja (PG) e de alguns atributos físicos (macoporosidade - MA, microporosidade - MI, porosidade total - PT e densidade do solo - DS) de um Latossolo Vermelho Distroférrico da Fazenda de Ensino, Pesquisa e Extensão - FE/ Unesp, Selvíria - MS.

\begin{tabular}{|c|c|c|c|c|c|c|c|c|c|c|c|c|}
\hline \multirow{2}{*}{ Atributos } & \multicolumn{12}{|c|}{ - } \\
\hline & PG & MA1 & MA2 & MA3 & MI1 & MI2 & MI3 & PT1 & PT2 & PT3d & DS1 & DS2 \\
\hline MA1 & $0,295^{* *}$ & - & - & - & - & - & - & - & - & - & - & - \\
\hline MA2 & $0,092^{\mathrm{NS}}$ & $0,558^{* *}$ & - & - & - & - & - & - & - & - & - & - \\
\hline MA3 & $0,262^{*}$ & $0,561^{* *}$ & $0,669^{* *}$ & - & - & - & - & - & - & - & - & - \\
\hline MI1 & $-0,202^{*}$ & $-0,539^{* *}$ & $-0,564^{* *}$ & $-0,515^{* *}$ & - & - & - & - & - & - & - & - \\
\hline MI2 & $-0,100^{\mathrm{NS}}$ & $-0,536^{* *}$ & $-0,641^{* *}$ & $-0,569^{* *}$ & $0,617^{* *}$ & - & - & - & - & - & - & - \\
\hline MI3 & $-0,152^{\mathrm{NS}}$ & $-0,488^{* *}$ & $-0,561^{* *}$ & $-0,607^{* *}$ & $0,510^{* *}$ & $0,643^{* *}$ & - & - & - & - & - & - \\
\hline PT1 & $0,037^{\mathrm{NS}}$ & $0,311^{* *}$ & $-0,021^{\mathrm{NS}}$ & $-0,017^{\mathrm{NS}}$ & $0,469^{* *}$ & $0,168^{\mathrm{NS}}$ & $0,179^{*}$ & - & - & - & - & - \\
\hline PT2 & $-0,086^{\mathrm{NS}}$ & $-0,044^{\mathrm{NS}}$ & $0,286^{*}$ & $0,024^{\mathrm{NS}}$ & $0,168^{\mathrm{NS}}$ & $0,426^{* *}$ & $0,236^{*}$ & $0,198^{*}$ & - & - & - & - \\
\hline PT3d & $0,059^{\mathrm{NS}}$ & $0,058^{\mathrm{NS}}$ & $0,160^{\mathrm{NS}}$ & $0,357^{* *}$ & $0,018^{\mathrm{NS}}$ & $0,024^{\mathrm{NS}}$ & $0,375^{* *}$ & $0,094^{\mathrm{NS}}$ & $0,291^{*}$ & - & - & - \\
\hline DS1 & $-0,221^{*}$ & $-0,377^{* *}$ & $-0,052^{\mathrm{NS}}$ & $-0,018^{\mathrm{NS}}$ & $-0,086^{\mathrm{NS}}$ & $-0,054^{\mathrm{NS}}$ & $0,072^{\mathrm{NS}}$ & $-0,452^{* *}$ & $-0,135^{\mathrm{NS}}$ & $0,068^{\mathrm{NS}}$ & - & - \\
\hline DS2 & $0,135^{\mathrm{NS}}$ & $0,079^{\mathrm{NS}}$ & $-0,089^{\mathrm{NS}}$ & $0,051^{\mathrm{NS}}$ & $-0,182^{*}$ & $-0,274^{*}$ & $-0,132^{\mathrm{NS}}$ & $-0,138^{\mathrm{NS}}$ & $-0,554^{* *}$ & $-0,082^{\mathrm{NS}}$ & $0,119^{\mathrm{NS}}$ & - \\
\hline DS3 & $0,060^{\mathrm{NS}}$ & $-0,123^{\mathrm{NS}}$ & $-0,096^{\mathrm{NS}}$ & $-0,260^{*}$ & $-0,021^{\mathrm{NS}}$ & $-0,158^{\mathrm{NS}}$ & $-0,143^{\mathrm{NS}}$ & $-0,157^{\mathrm{NS}}$ & $-0,292^{*}$ & $-0,384^{* *}$ & $0,094^{\mathrm{NS}}$ & $0,280^{*}$ \\
\hline
\end{tabular}

(A)*significativo a $5 \%$ de probabilidade; ** significativo a $1 \%$ de probabilidade; ns não significativo; camadas de 1,2 e 3 correspondem, respectivamente, a $0,0-0,10 ; 0,10-0,20$ e $0,20-0,30 \mathrm{~m}$.

$\mathrm{PG}=4,74 \cdot 10^{3}+1,74 \cdot 10^{-2^{*}} \cdot \mathrm{MA} 3+3,48 \cdot 10^{-1^{*}} \cdot \mathrm{DS} 1+3,64 \cdot 10^{-}$

${ }^{2 *} \cdot \operatorname{DS} 3\left(\mathrm{r}^{2}=0,221^{* *}\right)$

A análise geoestatística (Tabela 3 ) evidenciou excelentes semivariogramas, tanto para a planta, quanto para o solo. O melhor foi ajustado para a MA1, com um coeficiente de determinação espacial de 0,898 ; enquanto que, para a $\mathrm{PG}$, o semivariograma foi de 0,821 , superior ao obtido por REICHERT et al. (2008), com 0,708, também para soja, considerado moderado. Por outro lado, todos os atributos

Tabela 3 - Parâmetros dos semivariogramas (simples e cruzados) ajustados para a produtividade de grãos de soja (PG) e de alguns atributos físicos (macoporosidade - MA, microporosidade - MI, porosidade total - PT e densidade do solo - DS) de um Latossolo Vermelho Distroférrico da Fazenda de Ensino, Pesquisa e Extensão - FE/Unesp, Selvíria - MS.

\begin{tabular}{|c|c|c|c|c|c|c|c|c|c|}
\hline Atributo & modelo $^{(a)}$ & $\begin{array}{l}\text { efeito pepita } \\
\qquad\left(\mathrm{C}_{0}\right)\end{array}$ & $\begin{array}{c}\text { patamar } \\
\left(\mathrm{C}_{0}+\mathrm{C}\right)\end{array}$ & $\begin{array}{c}\text { variância } \\
\text { estrutural (C) }\end{array}$ & $\begin{array}{l}\text { alcance } \\
(\mathrm{m})\left(\mathrm{A}_{0}\right)\end{array}$ & $r^{2}$ & $\mathrm{SQR}^{(\mathrm{B})}$ & $\mathrm{ADE}^{(\mathrm{C})}$ & $\begin{array}{c}\text { classe de } \\
\text { dependência } \\
\text { espacial }\end{array}$ \\
\hline PG $\left(\mathrm{kg} \mathrm{ha}^{-1}\right)$ & gau. & $2,22.10^{5}$ & $8,57.10^{5}$ & $6,35 \cdot 10^{5}$ & 44,5 & 0,821 & $1,33 \cdot 10^{11}$ & 74,1 & moderada \\
\hline \multicolumn{10}{|l|}{$\gamma(\mathrm{h})$ simples do solo } \\
\hline $\operatorname{MA1}\left(\mathrm{m}^{3} \mathrm{~m}^{-3}\right)$ & gau. & $7,7.10^{-5}$ & $9,74.10^{-4}$ & $8,97 \cdot 10^{-4}$ & 63,9 & 0,898 & $2,05.10^{-7}$ & 92,1 & alta \\
\hline $\operatorname{MA} 2\left(\mathrm{~m}^{3} \mathrm{~m}^{-3}\right)$ & exp. & $1,2.10^{-4}$ & $9,00.10^{-4}$ & $7,80.10^{4}$ & 42,1 & 0,791 & $1,60.10^{-7}$ & 86,7 & alta \\
\hline $\operatorname{MA} 3\left(\mathrm{~m}^{3} \mathrm{~m}^{-3}\right)$ & esf. & $1.10^{-6}$ & $9,72.10^{-4}$ & $9,71.10^{-4}$ & 31,0 & 0,809 & $3,13 \cdot 10^{-7}$ & 99,9 & alta \\
\hline $\operatorname{MI} 1\left(\mathrm{~m}^{3} \mathrm{~m}^{-3}\right)$ & exp. & $1.10^{-6}$ & $1,19.10^{-3}$ & $1,19.10^{-3}$ & 42,9 & 0,792 & $5,71.10^{-7}$ & 99,9 & alta \\
\hline $\operatorname{MI} 2\left(\mathrm{~m}^{3} \mathrm{~m}^{-3}\right)$ & gau. & $1,03 \cdot 10^{-4}$ & $1,04 \cdot 10^{-3}$ & $9,33 \cdot 10^{-3}$ & 31,8 & 0,847 & $1,64 \cdot 10^{-7}$ & 90,1 & alta \\
\hline $\operatorname{MI} 3\left(\mathrm{~m}^{3} \mathrm{~m}^{-3}\right)$ & esf. & $1.10^{-6}$ & $8,72.10^{-4}$ & $8,71.10^{-4}$ & 32,5 & 0,836 & $1,26.10^{-7}$ & 99,9 & alta \\
\hline DS1 $\left(\mathrm{kg} \mathrm{dm}^{-3}\right)$ & epp. & $4,70.10^{-3}$ & $4,70.10^{-3}$ & - & - & - & - & - & - \\
\hline $\mathrm{DS} 2\left(\mathrm{~kg} \mathrm{dm}^{-3}\right)$ & epp. & $3,95 \cdot 10^{-3}$ & $3,95 \cdot 10^{-3}$ & - & - & - & - & - & - \\
\hline $\operatorname{DS} 3\left(\mathrm{~kg} \mathrm{dm}^{-3}\right)$ & gau. & $2,05.10^{-3}$ & $2,83.10^{-3}$ & $7,8.10^{-4}$ & 34,6 & 0,542 & $2,35.10^{-7}$ & 27,6 & moderada \\
\hline PT1 $\left(\mathrm{m}^{3} \mathrm{~m}^{-3}\right)$ & epp. & $6,30 \cdot 10^{-4}$ & $6,30 \cdot 10^{-4}$ & - & - & - & - & - & - \\
\hline PT2 $\left(\mathrm{m}^{3} \mathrm{~m}^{-3}\right)$ & epp. & $6,95 \cdot 10^{-4}$ & $6,95 \cdot 10^{-4}$ & - & - & - & - & - & - \\
\hline PT3 $\left(\mathrm{m}^{3} \mathrm{~m}^{-3}\right)$ & epp. & $6,40.10^{-4}$ & $6,40.10^{-4}$ & - & - & - & - & - & - \\
\hline $\mathrm{PG}=\mathrm{f}(\mathrm{MA} 1)\left(\mathrm{kg} \mathrm{ha}^{-1}\right)$ & gau. & $1,00.10^{-2}$ & $\begin{array}{l}\gamma(\mathrm{h}) \mathrm{cr} \\
8,68\end{array}$ & $\begin{array}{l}\text { do entre atrib } \\
8,67\end{array}$ & 38,6 & 0,553 & $1,24.10^{2}$ & 99,9 & alta \\
\hline $\mathrm{PG}=\mathrm{f}(\mathrm{MA} 2)\left(\mathrm{kg} \mathrm{ha}^{-1}\right)$ & gau. & $1,00.10^{-2}$ & 7,06 & 7,05 & 22,5 & 0,411 & $6,02.10$ & 99,9 & alta \\
\hline $\mathrm{PG}=\mathrm{f}(\mathrm{MI} 2)\left(\mathrm{kg} \mathrm{ha}^{-1}\right)$ & gau. & $-1,00.10^{-2}$ & $-1,184.10$ & $-1,183.10$ & 81,8 & 0,633 & $2,55.10^{2}$ & 99,9 & alta \\
\hline $\mathrm{MA} 2=\mathrm{f}(\mathrm{MI} 2)\left(\mathrm{m}^{3} \mathrm{~m}^{-3}\right)$ & gau. & $-1,00.10^{-6}$ & $-7,68.10^{-4}$ & $-7,67.10^{-4}$ & 35,5 & 0,897 & 1,25 & 99,9 & alta \\
\hline
\end{tabular}

(a) exp.=exponencial, gau=gaussiano, esf=esférico e epp.=efeito pepita puro; ${ }^{(b)} \mathrm{SQR}=$ soma dos quadrados dos resíduos; ${ }^{(c)} \mathrm{ADE}=\mathrm{avaliador}$ da dependência espacial; camadas de 1,2 e 3 correspondem, respectivamente, a 0,0-0,10;0,10-0,20 e 0,20-0,30m. 
pesquisados, com exceção das PT, DS1 e DS2, apresentaram dependência espacial (efeito pepita puro). Dessa forma, evidenciaram que suas distribuições no espaço não foram aleatórias, uma vez que a classe de dependência espacial da PG foi de 74,1\% (moderada), enquanto que para os atributos do solo variou entre 54,2\% (moderada/DS3) e 89,8\% (alta/MA1), concordando tal fato com os trabalhos de CARVALHO et al. (2002, 2003), SOUZA et al. (2004).

$\mathrm{Na}$ análise das cokrigagens (Tabela 3 ), o enfoque foi para a $\mathrm{PG}=\mathrm{f}(\mathrm{MA} 1)$, uma vez que este apresentou semivariograma cruzado com todos os lags distribuídos apenas em um dos quadrantes (Figura 1c), denotando um aspecto confiável entre as variáveis $\mathrm{PG}$ e MA1. Para a $\mathrm{PG}=\mathrm{f}(\mathrm{MI} 2)$, houve uma relação inversa entre suas variáveis. Assim, espacialmente avaliada, em suas krigagens simples, onde ocorreu uma elevação da microporosidade do solo na segunda camada, a produtividade foi reduzida. Em contrapartida, a $\mathrm{PG}=\mathrm{f}(\mathrm{MA} 2)$ seguiu a mesma tendência da MA1, ou seja, onde ocorreu aumento da macroporosidade do solo, tanto na primeira, quanto na segunda camada (regiões sudeste e sudoeste da Figura 1a e b), com valores entre $0,072-0,112\left(\mathrm{~m}^{3} \mathrm{~m}^{-3}\right)$, a PG variou entre $3249-4426 \mathrm{~kg} \mathrm{ha}^{-1}$. Desse modo, a tendência desses dados está de acordo com SANTOS et al. (2006), que apresentaram mapas de krigagens, indicando que, nas áreas de menor compactação do solo, a produtividade da planta foi maior e vice-versa.

Como a MA1 apresentou correlação direta e significativa com o PG (Tabela 2), a PG e a MA1 apresentaram ajustes semivariográficos simples (Tabela 3) e satisfatório ajuste da co-krigagem entre ambos (Figuras $1 \mathrm{a}, \mathrm{b}, \mathrm{d}$ ), pode-se inferir que a MA1 foi o melhor indicador da qualidade física do solo estudado, quando destinado a avaliar a produtividade de soja em sistema plantio direto, em solo de cerrado.

\section{CONCLUSÕES}

Os atributos estudados apresentaram variabilidade e seguiram padrões espaciais bem definidos, com alcances entre 31,0-63,9m. Espacialmente, houve correlação direta entre a $\mathrm{PG}$ e MA na camada de 0,0-0,10m e 0,10-0,20m. A macroporosidade do solo, avaliada na camada de 0 0,10m (MA1), apresentou-se como o melhor indicador da qualidade física do solo quando destinado à produtividade de soja em sistema plantio direto, em solo de cerrado.

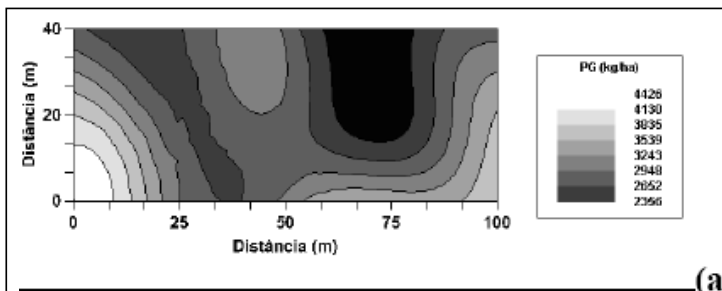

PG

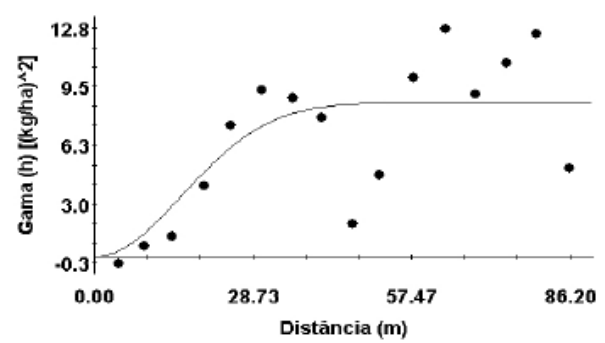

(c)

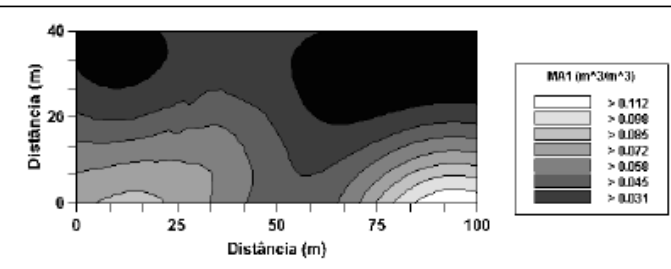

MA1

(b)

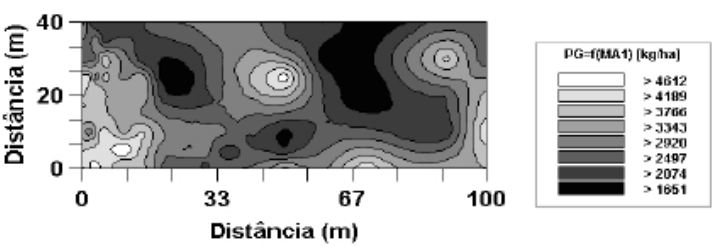

(d)

\section{$\mathbf{P G}=\mathbf{f}(\mathrm{MA1})$}

$\mathbf{P G}=\mathbf{f}(\mathrm{MA1})$

Figura 1 - Mapas de krigagem simples (a, b), semivariograma cruzado (c) e mapa da cokrigagem (d) da produtividade de grãos de soja (PG) e da macroporosidade superficial (MA1; 0-0,10m) de um Latossolo Vermelho Distroférrico de Selvíria (MS).

Ciência Rural, v.40, n.3, mar, 2010. 


\section{REFERÊNCIAS}

ABREU, S.L. et al. Variabilidade espacial de propriedades físicohídricas do solo, da produtividade e da qualidade de grãos de trigo em argissolo franco arenoso sob plantio direto. Cienc. Rural, v.33, n.2, p.275-282, 2003. Disponível em: <http:// www.scielo.br/scielo.php? script $=$ sci_abstract\&pid $=\mathrm{s} 0103$ $84782003000200015 \& \operatorname{lng}=\mathrm{pt} \& \mathrm{nrm}=\mathrm{iso} \& \operatorname{lng}=\mathrm{pt}>$. Acesso em: 20 nov. 2008. doi: 10.1590/s0103-84782003000200015.

CARVALHO, M.P. et al. Variabilidade espacial de atributos físicos em um Latossolo Vermelho distrófico sob preparo convencional em Selvíria, estado de Mato Grosso do Sul. Acta Sci Agron, v.24, p.1353-1361, 2002.

CARVALHO, M.P. et al. Variabilidade espacial de atributos de um solo sob videira em Vitória Brasil (SP). R Bras Ci Solo, v.27, p.695-703, 2003. Disponível em: $<$ http://www.scielo.br/scielo.php?pid=S0100$06832003000400014 \&$ script $=$ sci_abstract\&tlng $=\mathrm{pt}>$. Acesso em: 20 nov. 2008. doi: 10.1590/S0100-06832003000400014.

EMPRESA BRASILEIRA DE PESQUISA AGROPECUÁRIA EMBRAPA. Manual de métodos de análise do solo. 2.ed. Rio de Janeiro, 1997. (numeração capitulada).

EMPRESA BRASILEIRA DE PESQUISA AGROPECUÁRIA EMBRAPA. Sistema brasileiro de classificação de solos. 2.ed. Rio de Janeiro, 2006. 306p.

FAHL, J.I. et al. (Eds.). Instruções agrícolas para as principais culturas econômicas. Campinas: IAC, 1998. 396p. (Boletim 200).

FARACO, M.A. et al. Seleção de modelos de variabilidade espacial para elaboração de mapas temáticos de atributos físicos do solo e produtividade da soja. R Bras Ci Solo, v.32, p.463-476, 2008. disponível em: <http://www.scielo.br/scielo.php?pid=s0100$06832008000200001 \&$ script $=$ sci abstract\&tlng $=\mathrm{e}>$. Acesso em: 20 nov. 2008 . doi: $10.1590 / \mathrm{s} 0100-06832008000200001$.

FREDDI, O.S. et al. Produtividade do milho relacionada com a resistência mecânica à penetração do solo sob preparo convencional. Eng Agríc, v.26, p.113-121, 2006. Disponível em: $<$ http:// www.scielo.br/scielo.php? script $=$ sci_abstract\&pid=S0100$69162006000100013 \& \operatorname{lng}=\mathrm{pt} \& \mathrm{nrm}=\mathrm{iso} \& \mathrm{t} \operatorname{lng}=\mathrm{pt}>$. Acesso em: 20 nov. 2008. doi: 10.1590/S0100-69162006000100013.

GS+: Geostatistics for environmental sciences. 7.ed. Michigan, Plainwell: Gamma Desing Software, 2004. 159p.

JOHANN, J.A. et al. Variabilidade espacial dos atributos físicos do solo e da produtividade em um Latossolo Bruno distrófico da região de Cascavel, PR. Rev Bras Eng Agric Ambient, v.8, p.212-219, 2004. Disponível em: <http://www.scielo.br/
scieloOrg/php/cited Scielo.php?pid=S 1415 $43662004000200008 \&$ lang=pt $>$. Acesso em: 20 nov. 2008. doi: $10.1590 / \mathrm{S} 1415-43662004000200008$.

KIEHL, E.J. Manual de edafologia: relações solo-planta. Piracicaba: Agronômica Ceres, 1979. 264p.

MELO FILHO, J.F. et al. Análise estatística exploratória e variabilidade da densidade do solo em um perfil de Latossolo Amarelo coeso dos tabuleiros costeiros da Bahia. Ciênc Agrotec, v.30, p.199-205, 2006.

REICHERT, J.M. et al. Variabilidade espacial de Planossolo e produtividade de soja em várzea sistematizada: análise geostatística e análise de regressão. Cienc Rural, v.38, p.981-988, 2008. Disponível em: <http://www.scielo.br/scielo.php?pid=S0103$84782008000400012 \&$ script $=$ sci_abstract\&tlng $=$ pt $>$. Acesso em: 20 nov. 2008. doi: 10.1590/S0103-84782008000400012.

REICHERT, J.M. et al. Reference bulk density and critical degreeof-compactness for no-till crop production in subtropical highly weathered soils. Soil Till Res, v.102, p.242-254, 2009.

SANTOS, M.L. et al. Correlação linear e espacial entre produtividade de milho e atributos físicos de um Latossolo Vermelho distroférrico sob plantio direto do cerrado brasileiro. Acta Sci, v.28, p.:313-321, 2006. Disponível em: <http:// www.periodicos.uem.br/ojs/index.php/ActaSciAgron/article/ viewArticle/939>. Acesso em: 20 nov. 2008. doi:10.4025.

SCHLOTZHAVER, S.D.; LITTELL, R.C. SAS System for elementary statistical analysis. 2.ed. Cary, 1997. 905p.

SHAPIRO, S.S.; WILK, M.B. An analysis of variance test for normality: complete samples. Biometrika, v.52, p. 591-611, 1965.

SOARES, A. Geoestatística para as ciências da terra e do ambiente. Lisboa: Instituto Superior Técnico, 2000. 209p.

SOUZA, Z.M. et al. Variabilidade espacial de atributos físicos em um Latossolo Vermelho distrófico sob semeadura direta em Selvíria (MS). R bras Ci Solo, v.25, p.:699-707, 2001.

SOUZA, Z. M. et al. Variabilidade espacial de atributos físicos de um latossolo vermelho sob cultivo de cana-de-açúcar. $\mathbf{R}$ bras Eng Agríc Amb, v.8, p.51-58, 2004.

TORMENA, C.A. et al. Propriedades físicas do solo sob plantio direto influenciadas por calagem, preparo inicial e tráfego. $\mathbf{R}$ Bras Ci Solo, v.22, p.301-309, 1998.

TRANGMAR, B.B; et al. Application of geostatistics to spatial studies of soil properties. Adv Agron, v.38, p.45-94, 1985. 